\title{
Screening of Early Warning Parameters of Dengue Fever Syndrome in Mengla County Based on Space- Time Rearrangement Scanning Statistics
}

\section{Rongyan $\mathrm{Gu}$}

Kunming medical university

\section{Shen Huang}

Kunming medical university

Ling Zhang

Kunming medical university

Wei Liu(D liuweikm@qq.com )

Kunming medical university

Yan Li

Kunming Medical University

\section{Research Article}

Keywords: dengue fever, space-time rearrangement scan, space-time aggregation, parameter filtering, symptom monitoring

Posted Date: December 29th, 2020

DOI: https://doi.org/10.21203/rs.3.rs-135059/v1

License: (c) (1) This work is licensed under a Creative Commons Attribution 4.0 International License. Read Full License 


\section{Abstract}

Objective: The objective of this study was to establish the combination of time and space parameters for spatiotemporal rearrangement scanning. The spatial and temporal rearrangement of dengue cluster events was performed by using dengue syndrome to evaluate the effects of different spatiotemporal parameter combinations on the early warning effect. This study aimed to select the best spatiotemporal parameter combination for early warning of dengue syndrome and establish a perfect early warning model of dengue syndrome based on spatiotemporal rearrangement scan statistics.

Methods: The data of dengue syndrome in eighty medical institutions (including township hospitals and village clinics) in the symptom monitoring system of medical institutions in Mengla County in 2017 and the data of reported cases of dengue in the direct reporting system of infectious diseases were collected using Excel to describe the time, region and population distribution of dengue fever. The Java server page was scanned to establish different spatiotemporal parameter combinations $(X, Y)$. $X$ was the time parameter, and $Y$ was the space parameter. The maximum number of early warning days was set to four, and when including the date of the gathering event, a total of five days. The dengue fever clusters in Mengla County in 2017 were scanned day by day in time and space, and the sensitivity and the number of days of advance warning were used as the warning indicators to comprehensively evaluate the warning effects of nine warning schemes.

Results: 1. A total of two hundred forty-seven cases of dengue fever were reported in Mengla County in 2017 , with an incidence rate of $85.79 / 100,000$. The peak incidence was mainly from August to November; cases of dengue fever reported by the infectious disease direct reporting system were distributed over eight medical institutions. Mengla County People's Hospital had the largest number of reported cases; the ratio of males to females was roughly balanced (approximately 1:1). The largest population distribution was 100 cases in farmers (40.49\%), followed by 50 cases of business service personnel (20.24\%). 2. Nine different spatiotemporal parameter combinations were used to scan a total of 225 times, which were performed for five dengue fever clusters in Mengla County in 2017. The aggregation region, LLR(Log Likelihood Ratio), RR and $P$ values of the warning under each scan combination were obtained. 3. Sensitivity analysis of nine parameter combinations for five dengue aggregation events included the following: the $(3,10),(4,10),(5,10),(4,15),(5,15)$ combination alerted to five dengue aggregation events, and the sensitivity was $100 \%$; the $(3,15)$ combination alerted to four dengue aggregation events, and the sensitivity was $80 \%$; the $(3,5),(4,5),(5,5)$ combination alerted to three dengue aggregation events, and the sensitivity was $60 \% .4$. Analysis of the early warning times of five dengue aggregation events by nine parameter combinations resulted in the following: the $(3,10)$ combination early warning times of five dengue aggregation events were three days, four days, four days, one day and one day, with an average early warning time of two point six days; the $(4,10),(5,10)$ combination early warning times of five dengue aggregation events were two days, three days, four days, one days and zero day, respectively, with an average early warning time of two days; the $(3,15)$ combination early warning times of five dengue aggregation events were zero day, four days, four days, one day and one day, with an average early warning time of two days; the $(5,15)$ combination early warning times of five dengue 
aggregation events were one day, four days, four days, one day and zero day, with an average early warning time of 2 days; the $(4,5),(5,5),(4,15)$ combination early warning times of five dengue aggregation events were zero day, four days, three days, one day and zero day, with an average early warning time of one point six days; the $(3,5)$ combination early warning times of five dengue aggregation events were one day, four days, three days, zero day and zero day, with an average early warning time of one point six days. 5 . Analysis of the duration warning time of five dengue aggregation events by nine parameter combinations included the following: for the $(3,10)$ combination among the five dengue aggregation events, the average duration warning days was two point two days; for the $(4,10),(5,10),(5$, 15) combination among the five dengue aggregation events; the average duration warning days was two days, and the average duration warning days of other parameter combinations were less than two days.

Conclusions: 1. Dengue fever has an obvious seasonal trend in Mengla County, and the cases are mainly concentrated in summer and autumn. In this time period, we should strengthen the corresponding prevention and control of dengue fever, especially the main gathering areas of farmers and commercial service groups. 2. Based on the data of dengue syndrome, time-space rearrangement scanning of dengue clustering events in Mengla County was carried out, and the optimal time-space clustering combination was selected. In the future, the combination of the $(3,10)$ parameters can be preferentially used for the early warning of dengue outbreaks in this area by using dengue syndrome. In addition, the combination of the $(4,10),(5,10)$, and $(5,15)$ parameters can be used as an alternative parameter combination. 3 . In the future, when the optimal parameter combination is used for the early warning of dengue fever, a graded response can be adopted according to the actual situation, which maximally ensures that real aggregation events are detected while avoiding excessive waste of resources.

\section{Background}

Dengue fever (DF) is an acute infectious disease caused by the dengue virus and transmitted by the bites of Aedes aegypti and Aedes albopictus ${ }^{[1]}$. The clinical symptoms are raised fever, muscle, bone, and joint pain, skin rash, lymphadenopathy and leukopenia. The incubation period is 3-14 days, with an average of 7 days. The disease has the characteristics of universal susceptibility, rapid spread, high incidence, and high mortality of severe types. It is widely prevalent in tropical and subtropical regions and has become a serious global public health problem ${ }^{[2-4]}$. After the outbreak in 2013, there was another epidemic outbreak again in 2016 and 2017, and the number of cases rose from 44 in 2013 to 247 in 2017 in the area. The number of cases doubled, and the situation of dengue fever prevention and control in Mengla County was very severe ${ }^{[5]}$. Therefore, it is necessary to establish a fast and effective dengue fever prediction and early warning system in Mengla County.

Kulldorff et al. proposed forward-looking spatiotemporal scanning statistics to detect spatiotemporal aggregation patterns of infectious diseases in $2001^{[6-8]}$. This model does not rely on population data, and it can make predictions based on case data, which can provide advantages for early warning of infectious diseases and cluster detection of areas with missing population data ${ }^{[9]}$. When using 
spatiotemporal rearrangement scan statistics to detect the aggregation of infectious diseases, the setting of parameters is crucial to the scan window. In previous studies, it is generally specified that the maximum spatial scanning window is $50 \%$ of the total at-risk population, and the longest scanning period is 7 days $^{[10-11]}$. However, different diseases have their own outbreak characteristics, and the range of spatiotemporal scanning should vary from disease to disease. For example, Ailiyun et al. ${ }^{[12]}$ found that in the detection of dengue fever spatiotemporal clustering, the maximum scanning window for the total epidemic is $50 \%$ risk populations of the disease, $30 \%$ for sporadic epidemics; Tangxianyan et al ${ }^{[13]}$ took $5 \%, 10 \%, 15 \%, 20 \%, 25 \%, 30 \%, 35 \%, 40 \%, 45 \%$, and $50 \%$ of the total risk population to detect and analysed liver cancer in Guangxi, and they recommend an at-risk population in the range of $20-30 \%$ as the maximum spatial scanning window. Coulstion ${ }^{[14]}$ considered the differences in terrains and carried out spatial scanning within $30 \%$ of the at-risk population, and detected small areas with linear distribution; Xiao Yanyan ${ }^{[15]}$ used spatiotemporal rearrangement scan statistics to conduct research on parameter screening for hand-foot-mouth disease outbreak early warning. This is the first time that spatiotemporal rearrangement scans have been used to adjust the parameters of the time range and space range at the same time and to compare different parameter values of early warning effects in China.

In order to improve the early warning capability of infectious disease outbreaks or epidemics in China's border areas, the collaborative innovation of disease prevention and control in Yunnan Province launched the project of "Application Project of Symptom Monitoring, Early Warning, Prevention and Control System in Border Counties in Yunnan Province"(Fig. 1), on January 12, 2014. Based on the project, this study conducted a spatiotemporal rearrangement scan of dengue fever syndromes of dengue fever clusters that occurred in Mengla County in 2017 and established alternative time parameters and time parameters for the spatiotemporal rearrangement scan. Spatial parameter combinations evaluate the influence of different time parameter and spatial parameter combinations on the early warning effect, screen out the optimal spatiotemporal parameter combination suitable for early warning of dengue fever syndrome, and establish a perfect dengue syndrome early warning model based on the amount of time and space rearrangement scans.

\section{Data}

To further improve the monitoring, prevention and control of infectious diseases in the border areas of Yunnan Province, enrich the current public health emergency early warning system, and protect the public health safety of the Greater Mekong countries in the region, we independently developed and established the "Symptoms of Yunnan Province Border Areas Monitoring System". The system was launched on August 7, 2015, in the three border ports of Yunnan Province, Mengla County, Ruili City, and Yingjiang County. It mainly includes the symptom monitoring system for medical institutions, the monitoring system for elementary school student absenteeism, and the monitoring system for fever patients in port inspection and quarantine institutions. By monitoring 85 kinds of clinical symptoms in border areas, early warning of the outbreak of 18 target infectious diseases can be achieved. 
The data come from the Symptom Monitoring System and Network Infectious Disease Reporting Information System of Mengla County Medical Institutions. Since the symptom monitoring system was officially launched in January 2016, it was found in a preliminary study ${ }^{[16]}$ that in the first half of 2016, the quality of data reporting was unstable. Since July, the data reporting rate of monitoring points in Mengla has stabilized at more than $60 \%$; since October, the data began to stabilize at more than $70 \%$. As the project continues, the quality of data reporting is rising. Therefore, the specific data selected for this study are the dengue fever syndrome data from 80 medical institutions (including township health centres and village clinics) in the medical institution symptom monitoring system in 2017 and the actual reported cases of dengue fever in the infectious disease direct reporting system. The actual dengue fever gathering incidents are summarized.

\section{Method}

\subsection{Spatiotemporal rearrangement scan statistics}

This sDistribution of Dengue Fever Accumulation Events in Mengla tudy used spatiotemporal rearrangement scan statistics ${ }^{[9]}$. The basic principle was to randomly select a geographic location point in a certain predetermined research area, use this point as the centre of the cylindrical scanning window, the radius of the bottom surface of the cylinder as the spatial distance, and the height of the cylinder as the time range, constantly facing the bottom surface. The radius and height of the circle are changed (that is, the spatial area and time range covered by the cylindrical window are constantly changing) until the upper limit of the predetermined range is reached. This process was repeated continuously, and scanning windows were available for all positions in the study area. Each scanning window calculated the corresponding theoretical number of incidences based on the actual number of incidences (number of symptoms), and the corresponding LLR can be calculated using the actual number of incidences inside and outside each scanning window and the number of theoretical incidences. According to LLR, the abnormal degree and aggregation of the disease can be detected. To avoid false positives in multiple windows, the largest LLR was tested using Monte Carlo statistical randomization ${ }^{[17-18]}$. The Monte Carlo method was used to generate a series of randomly distributed simulation data sets. Each data set had a simulated maximum LLR. The maximum LLR of real data was compared with the maximum LLR of some columns of simulated data, and the corresponding maximum LLR of real data was calculated. The $P$ value was used to accurately provide early warning signals. The smaller the $P$ value, the less likely the number of cases in the window corresponding to the maximum LLR of the real data was randomly distributed, and the higher the aggregation. For example, the real largest LLR was ranked 50th, and its $P$ value $=50 /(999+1)=0.05$. If the ranking is the top 10 , its $P$ value $=10 /(999+1)=0.01$. The test level of this study was $P=0.05$.

2.2 Selection of time and space parameters

\subsubsection{Time parameters}


The height of the cylindrical scanning area is the time dimension, and different time parameters can change the size of the time scanning window. The current time parameter range is 1-7 days and cannot exceed 7 days. If it exceeds 7 days, an early warning cannot be reached. However, if the scan range is 12 days, the number of cases may be too small, and some cases may be missed; if the scan range is 6-7 days, abnormal clusters within the maximum time span can be gathered, but the earliest warning is not possible at this time, and the detection of the outbreak may be delayed. To accumulate enough cases of dengue fever syndrome and achieve early warning, the time parameters selected in this study were 3,4 , and 5 days.

\subsubsection{Spatial parameters}

The radius of the bottom circle of the cylindrical scanning area is the spatial dimension, and different time parameters can change the size of the time scanning window. In previous studies, the maximum spatial scanning window did not exceed $50 \%$ of the total population at risk. If the scanning range is too large, too many early warning signals will appear, leading to an increase in false positives, resulting in a waste of resources; if the scanning range is too small, the aggregation of some smaller areas may be ignored. In our study, the research site in Mengla County is too small, with a total area of $7056 \mathrm{~km}^{2}$ and a length of 84 kilometres; it includes 14 towns, and the total permanent population is 287,900 . Moreover, the data on dengue fever syndrome were gathered at village medical institutions, and a portion was located in village clinics. Therefore, the spatial scanning radius set in this study was set to $5 \mathrm{~km}, 10 \mathrm{~km}$, and $15 \mathrm{~km}$ in sequence.

\subsubsection{Spatiotemporal parameter combination}

The time parameters set in our study were 3 days, 4 days, and 5 days, and the spatial scanning radii were $5 \mathrm{~km}, 10 \mathrm{~km}$, and $15 \mathrm{~km}$. The parameter combinations are as follows. There are 9 combinations, namely, 9 early warning schemes.

Table 1 Spatiotemporal parameter combinations

\begin{tabular}{|llll|}
\hline Spatial parameters $Y(\mathrm{~km})$ & \multicolumn{3}{c|}{ Time parameter X (d) } \\
\cline { 2 - 4 } & 3 & 4 & 5 \\
\hline 5 & $\varangle 3,5 \rrbracket$ & $\varangle 4,5 \rrbracket$ & $\square 5,5 \rrbracket$ \\
\hline 10 & $\varangle 3,10 \rrbracket$ & $\varangle 4,10 \rrbracket$ & $(5,10)$ \\
\hline 15 & $(3,15)$ & $(4,15)$ & $(5,15)$ \\
\hline
\end{tabular}

\subsubsection{Sensitivity}

Sensitivity is the percentage of actual patients who were correctly judged as positive, according to the standard of the screening test. It can reflect the ability of a certain screening test to find patients. In a 
monitoring system whose purpose is early warning, sensitivity refers to the proportion of real outbreaks detected as outbreaks in the early warning system, and it is an important indicator for evaluating the early warning effect of the model ${ }^{[19]}$. In our study, the actual dengue fever event in 2017 was used as a reference, and different parameter combinations were used to warn of the actual dengue fever event. The sensitivity refers to the percentage of dengue fever events predicted by the model in the total true dengue fever events that occurred in 2017. The higher the sensitivity, the higher the accuracy is, that is, the stronger the model's ability to find clustered events. The calculation method is as follows:

\section{Sensitivit $y=\frac{\text { The number of dengue fever events predicted by the model }}{\text { Actual number of dengue fever clusters }}$}

\subsubsection{Days of Early Warning}

If the outbreak of the disease can be predicted in advance, the spread of the disease can be controlled in time, the incidence rate can be reduced, and the primary prevention and control of the disease can be effectively achieved. Therefore, this study also included the actual number of days of early warning as an evaluation indicator for the combination of different parameters. If the number of days for early warning is earlier, the early warning effect of parameter combination simulation is better and vice versa. The incubation period of dengue fever is 3-14 days, with an average incubation period of 7 days. It is generally believed that an effective early warning is half of the incubation period. Due to regional limitations, if the early warning days are too long, some other unrelated cases may be included, and the number of false positives will increase. Therefore, the maximum number of early warning days included in this study was 4 days, that is, based on the actual dengue fever gathering date, assuming that the date of a dengue fever gathering event is January 5,2017 , the scanning date was pushed forward 4 days in turn, and the scan dates included January 5, January 4, January 3, January 2, and January 1 . After more than 4 days, regardless of whether there is an early warning signal, the scan stopped; for each gathering event, 9 parameter combinations were used to scan 5 times each, and a total of 45 scans were obtained. Assuming a total of $\mathrm{N}$ aggregation events occurred in 2017 , the total number of scans is $45^{\star} \mathrm{N}$.

\subsection{Java server page scan window (scan tool)}

Java server pages (JSPs) are a dynamic web technology built by Sun Microsystems and other companies. This technology can insert Java program segments and JSP tags into traditional web page HTML files to form JSP files ( ${ }^{*}$.jsP) ${ }^{[20]}$, and the web applications developed by this can run on different operating systems, such as Android, iOS, and Windows, and web page logic can be separated from the web page design and display, making it faster and more convenient to use. The symptom monitoring and early warning system in our study is based on this technology, and window scanning was performed through Java server pages.

Java server page scanning steps: 
1. Figure 1 shows the web interface of the symptom monitoring system. After clicking on the timespace scanning model analysis above, you can enter the time-space scanning window.

2. In the data selection dialogue box that appears on Fig. 2, you can select symptoms, syndromes, prediction methods, convergence methods, end time, maximum time cluster, maximum radius, inspection level, etc. In this study, the dengue fever syndrome group was selected to gather at the medical institution by week, that is, to locate the medical institution; the maximum time cluster was set to 7 days, but due to the short period of 1-2 days, the cumulative number of cases may be too small; at 6-7 days, the scan window span is too large, and the detected outbreak may be delayed. Therefore, the maximum time cluster in this study does not consider the above two time ranges. The maximum radius is $15 \mathrm{~km}$; the inspection level is $\mathrm{P}=0.05$.

3. After selecting the data, the "Start Spatiotemporal Analysis" button was clicked to obtain a 7-week spatiotemporal analysis chart. The 7 weeks is based on the "end time" selected earlier and is obtained by forwarding 49 days in turn. If the end time is December 31, 2016, you can obtain 7 maps that are pushed forward 49 days (7 weeks) based on December 31. The numbers 1-10 appearing in each map represent the size of a possible disease outbreak. The smaller the number, the larger the LLR, and the smaller the $P$, the greater the possibility of an outbreak. Since the inspection level set in our study was 0.05 , if there are clusters with $P<0.05$, the numbers were displayed in red, and an early warning signal can be issued. At this time, based on the date of the red early warning number, the scan date was pushed forward in turn 1 day, stop until the warning signal was scanned. For example, if the red number 1 is scanned on November 19,2016, the gathering area is $A$, and the scanning date is pushed forward by one day, that is, November 18; if there is an early warning on the 18th, the gathering area is also $\mathrm{A}$, and it continues to be pushed forward to the 17th. If there is no warning signal on the 17th, the scanning will stop. It can be seen that the earliest warning for this gathering is November 18. Different parameter combinations can be used for scanning afterwards.

\section{Results}

3.1 Distribution of Dengue Fever Accumulation Events in Mengla County in 2017

The distribution of dengue fever cases from May to December 2017 is described by day. The epidemic lasted for 134 days, the county's emergency response and treatment were 141 days, and a total of 5 dengue fever clusters occurred. See Table 3 and Figure 3 for details.

Table 2 Temporal and spatial distribution of dengue fever events in 2017 


\begin{tabular}{|llll|}
\hline $\begin{array}{l}\text { Gathering } \\
\text { event }\end{array}$ & $\begin{array}{l}\text { First } \\
\text { gathering } \\
\text { time }\end{array}$ & Gathering institution & $\begin{array}{c}\text { Dengue } \\
\text { fever } \\
\text { cases }\end{array}$ \\
\hline 1 & $7 / 27$ & Mengla People's Hospital & 4 \\
\hline 2 & $8 / 28$ & Mengla People's Hospital & 5 \\
\hline 3 & $9 / 16$ & Mengla People's Hospital & 6 \\
\hline 4 & $10 / 14$ & $\begin{array}{l}\text { The Second Hospital of Mengla, Mengla People's Hospital, } \\
\text { Menglun Town Central Health Center }\end{array}$ & 9 \\
\hline 5 & $11 / 4$ & Mengla People's Hospital & 4 \\
\hline
\end{tabular}

Fig.3Daily distribution of dengue fever cases

3.2 Scanning and analysis of cluster events using different combinations of time and space parameters of dengue fever syndrome

There were 9 different spatiotemporal parameter combinations in our study, and the maximum number of early warning days was 4 days, including the date of the gathering event for 5 days. A total of 225 scans of five dengue fever gathering events occurred in Mengla County in 2017.

3.3 Time and space scanned maps of 5 dengue fever clusters(Taking the first dengue fever aggregation event on July 27, 2017 as an example, early warning analysis based on different spatiotemporal parameters of dengue syndrome).

The first dengue fever aggregation event occurred in the people's Hospital of Mengla County on July 27 was predicted by using the combination of nine spatiotemporal parameters, The results showed that: $(4,5),(5,5),(3,15)$ combinations did not scan dengue syndrome clustering within 4 days (including the day of aggregation event) of dengue fever cluster $(P>0.05)$; The other combinations could scan the aggregation of dengue syndrome $(P<0.05)$;After the $(3,10)$ combined scanning, the first clustering of dengue syndrome occurred on July 25 , the LLR value of internal and external of the aggregation area was 4.26 , and the RR value of incidence was 4.41. The gathering area was the second hospital of Mengla County and the Chengzi clinic of Mengla County, which was given early warning two days in advance; $(3,5),(4,10),(5,10),(5,15)$ combined scanning, the first gathering of dengue syndrome occurred on July 26. The LLR value and RR value inside and outside the gathering area were 4.17 and 4.27 , respectively. The gathering area was Chengzi clinic of Mengla,and the early warning was given one day in advance; $(4,15)$ combined scanning showed that the first gathering of dengue syndrome occurred on July 27 , with the LLR value of 4.08 and RR value of 4.19 . The gathering area was Chengzi clinic of Mengla. Early warning appeared on the day of dengue fever aggregation, 0 days in advance( Fig. 4, table 3 and table 4).

Table 3 Spatiotemporal aggregation analysis of different parameter combinations of the first aggregation event on July 27, 2017 


\begin{tabular}{|c|c|c|c|c|c|}
\hline $\begin{array}{l}\text { Spatiotemporal parameter } \\
\text { combination }\end{array}$ & $\begin{array}{l}\text { First warning } \\
\text { time(d) }\end{array}$ & Gathering area & LLR & RR & $P$ \\
\hline$\varangle 3,5 \rrbracket$ & $7 / 26$ & Chengzi clinic ,Mengla & 4.17 & 4.27 & $<0.01$ \\
\hline$₫ 4,5 \rrbracket$ & - & - & - & - & $>0.05$ \\
\hline$\otimes 5,5 \rrbracket$ & - & - & - & - & $>0.05$ \\
\hline \multirow[t]{2}{*}{$\varangle 3,10 \rrbracket$} & $7 / 25$ & Chengzi clinic Mengla & 4.26 & 4.41 & $<0.01$ \\
\hline & & $\begin{array}{l}\text { The second } \\
\text { hospital,Mengla }\end{array}$ & & & \\
\hline$\varangle 4,10 \bigotimes$ & $7 / 26$ & Chengzi clinic ,Mengla & 4.17 & 4.27 & $<0.01$ \\
\hline$\varangle 5,10 \bigotimes$ & $7 / 26$ & Chengzi clinic ,Mengla & 4.17 & 4.27 & $<0.01$ \\
\hline$\varangle 3,15 \rrbracket$ & - & - & - & - & $>0.05$ \\
\hline$\varangle 4,15 \rrbracket$ & $7 / 27$ & Chengzi clinic ,Mengla & 4.08 & 4.19 & $<0.01$ \\
\hline$\varangle 5,15 \rrbracket$ & $7 / 26$ & Chengzi clinic ,Mengla & 4.17 & 4.27 & $<0.01$ \\
\hline
\end{tabular}

Table 4 Early warning analysis of different parameter combinations of the first aggregation event on July 27,2017

\begin{tabular}{|c|c|c|c|}
\hline $\begin{array}{l}\text { Spatiotemporal parameter } \\
\text { combination }\end{array}$ & $\begin{array}{l}\text { Early warning } \\
\text { days } \square \text { ) }\end{array}$ & $\begin{array}{l}\text { Continuous warning } \\
\text { days }\end{array}$ & $\begin{array}{l}\text { Number of } \\
\text { warnings }\end{array}$ \\
\hline$\otimes 3,5 \rrbracket$ & 1 & 2 & 2 \\
\hline$\varangle 4,5 \rrbracket$ & 0 & 0 & 0 \\
\hline$\otimes 5,5 \rrbracket$ & 0 & 0 & 0 \\
\hline$\varangle 3,10 \bigotimes$ & 2 & 2 & 2 \\
\hline$\varangle 4,10 \bigotimes$ & 1 & 2 & 2 \\
\hline$\varangle 5,10 \bigotimes$ & 1 & 2 & 2 \\
\hline$\varangle 3,15 \rrbracket$ & 0 & 0 & 0 \\
\hline$\varangle 4,15 \rrbracket$ & 0 & 1 & 1 \\
\hline$₫ 5,15 \rrbracket$ & 1 & 2 & 2 \\
\hline
\end{tabular}

The analysis details of the remaining four cluster incidents are presented in the attachment(Fig.5-Fig.8).

3.4 Sensitivity analysis of 9 parameter combinations to 5 dengue fever cluster events 
The $(3,10),(4,10),(5,10),(4,15)$, and $(5,15)$ combination all forewarned of 5 dengue fever events, with a sensitivity of $100 \%$; the $(3,15)$ combination for early warning of 4 dengue fever events had a sensitivity of $80 \%$; the $(3,5),(4,5)$, and $(5,5)$ combination for early warning of 3 dengue fever events had a sensitivity of $60 \%$.

Table 5 Early warning sensitivity analysis of 9 spatiotemporal parameter combinations

\begin{tabular}{|c|c|c|c|}
\hline $\begin{array}{l}\text { Spatiotemporal } \\
\text { parameter combination }\end{array}$ & $\begin{array}{l}\text { The number of real events in } \\
\text { the model early warning }\end{array}$ & $\begin{array}{l}\text { Number of true } \\
\text { gathering events }\end{array}$ & Sensitivity[\%] \\
\hline$\varangle 3,5 \rrbracket$ & 3 & 5 & 60 \\
\hline$\varangle 4,5 \rrbracket$ & 3 & 5 & 60 \\
\hline$₫ 5,5 \rrbracket$ & 3 & 5 & 60 \\
\hline$\varangle 3,10 \rrbracket$ & 5 & 5 & 100 \\
\hline$\otimes 4,10 \rrbracket$ & 5 & 5 & 100 \\
\hline$₫ 5,10 \rrbracket$ & 5 & 5 & 100 \\
\hline$\varangle 3,15 \rrbracket$ & 4 & 5 & 80 \\
\hline$₫ 4,15 \rrbracket$ & 5 & 5 & 100 \\
\hline$\triangle 5,15 \rrbracket$ & 5 & 5 & 100 \\
\hline
\end{tabular}

3.5 Analysis of the early warning time of 5 dengue fever clusters with 9 parameter combinations

For the $(3,10)$ combination for 5 dengue fever clusters, early warnings were $3,4,4,1$, and 1 days in advance, with an average early warning time of 2.6 days; for the $(4,10),(5,10)$ combination for 5 dengue fever events in the cluster events, the early warnings were $2,3,4,1$, and 0 days in advance, and the average early warning time was 2 days; the $(3,15)$ combination had early warnings $0,4,4,1$, and 1 day in advance, and the average early warning time was 2 days; the $(5,15)$ combination had early warnings of 1 , $4,4,1$, and 0 day, and the average early warning time was 2 days; the $(4,5),(5,5),(4,15)$ combinations all had early warnings of $0,4,3,1$, and 0 days, with an average early warning time of 1.6 days; the $(3,5)$ combinations had $1,4,3,0$, and 0 days in advance, with an average early warning time of 1.6 days. See Table 5 for details.

Table 6 Early warning time for 9 combinations of time and space parameters 


\begin{tabular}{|c|c|c|c|c|c|c|}
\hline \multirow{2}{*}{$\begin{array}{l}\text { Spatiotemporal } \\
\text { parameter } \\
\text { combination }\end{array}$} & \multicolumn{6}{|c|}{ Early warning time(d) } \\
\hline & $\begin{array}{l}\text { Gathering } \\
\text { event } 1\end{array}$ & $\begin{array}{l}\text { Gathering } \\
\text { event } 2\end{array}$ & $\begin{array}{l}\text { Gathering } \\
\text { event } 3\end{array}$ & $\begin{array}{l}\text { Gathering } \\
\text { event } 4\end{array}$ & $\begin{array}{l}\text { Gathering } \\
\text { event } 5\end{array}$ & $\begin{array}{l}\text { Average } \\
\text { early } \\
\text { warning } \\
\text { time }\end{array}$ \\
\hline$\varangle 3,5 \rrbracket$ & 1 & 4 & 3 & 0 & 0 & 1.6 \\
\hline$₫ 4,5 \rrbracket$ & 0 & 4 & 3 & 1 & 0 & 1.6 \\
\hline$₫ 5,5 \rrbracket$ & 0 & 4 & 3 & 1 & 0 & 1.6 \\
\hline$\otimes 3,10 \rrbracket$ & 3 & 4 & 4 & 1 & 1 & 2.6 \\
\hline$\otimes 4,10 \rrbracket$ & 2 & 3 & 4 & 1 & 0 & 2.0 \\
\hline$\otimes 5,10 \bigotimes$ & 2 & 3 & 4 & 1 & 0 & 2.0 \\
\hline$\varangle 3,15 \rrbracket$ & 0 & 4 & 4 & 1 & 1 & 2.0 \\
\hline$₫ 4,15 \rrbracket$ & 0 & 4 & 3 & 1 & 0 & 1.6 \\
\hline$₫ 5,15 \rrbracket$ & 1 & 4 & 4 & 1 & 0 & 2.0 \\
\hline
\end{tabular}

3.6 Analysis of the continuous warning time of 5 dengue fever clusters with 9 parameter combinations

For the $(3,10)$ combination among the 5 dengue fever clusters, the average number of continuous warning days was 2.2 days; the average number of continuous warning days for the combinations of ( 4 , $10),(5,10)$, and $(5,15)$ was 2 days; the rest of the average continuous warning days of the parameter combination was less than 2 days (Table 6).

Table 7 The duration of the 9 spatiotemporal parameter combinations 


\begin{tabular}{|c|c|c|c|c|c|c|}
\hline \multirow{2}{*}{$\begin{array}{l}\text { Spatiotemporal } \\
\text { parameter } \\
\text { combination }\end{array}$} & \multicolumn{6}{|c|}{ Early warning time(d) } \\
\hline & $\begin{array}{l}\text { Gathering } \\
\text { event } 1\end{array}$ & $\begin{array}{l}\text { Gathering } \\
\text { event } 2\end{array}$ & $\begin{array}{l}\text { Gathering } \\
\text { event } 3\end{array}$ & $\begin{array}{l}\text { Gathering } \\
\text { event } 4\end{array}$ & $\begin{array}{l}\text { Gathering } \\
\text { event } 5\end{array}$ & $\begin{array}{l}\text { Average } \\
\text { early } \\
\text { warning } \\
\text { time }\end{array}$ \\
\hline$\varangle 3,5 \rrbracket$ & 2 & 3 & 2 & 0 & 0 & 1.4 \\
\hline$₫ 4,5 \rrbracket$ & 0 & 3 & 2 & 1 & 0 & 1.2 \\
\hline$₫ 5,5 \rrbracket$ & 0 & 3 & 2 & 1 & 0 & 1.2 \\
\hline$\varangle 3,10 \rrbracket$ & 2 & 3 & 3 & 1 & 2 & 2.2 \\
\hline$\varangle 4,10 \bigotimes$ & 2 & 3 & 3 & 1 & 1 & 2.0 \\
\hline$\otimes 5,10 \bigotimes$ & 2 & 3 & 3 & 1 & 1 & 2.0 \\
\hline$\varangle 3,15 \rrbracket$ & 0 & 3 & 3 & 1 & 2 & 1.8 \\
\hline$\varangle 4,15 \rrbracket$ & 1 & 2 & 2 & 1 & 1 & 1.4 \\
\hline$\varangle 5,15 \rrbracket$ & 2 & 3 & 3 & 1 & 1 & 2.0 \\
\hline
\end{tabular}

\section{Discussion}

4.1 Comprehensive evaluation and analysis of the early warning effect of different parameter combinations

In previous studies, Chen Wu et al. ${ }^{[21]}$ used fever symptoms to predict the early outbreak of dengue fever and found that in addition to fever symptoms, many patients also have symptoms such as skin rash, muscle pain, and headache. Only fever was used. Symptoms can lead to incomplete monitoring and incomplete data collection. Our study uses dengue fever syndrome data and 9 different spatiotemporal parameter combinations to conduct early warning analysis of 5 dengue fever cluster events that occurred in 2017. The syndrome data are more stable and comprehensive than single symptom data. Through further comparison and analysis of the sensitivity of 9 spatiotemporal parameter combinations, it was found that the combinations of $(3,10),(4,10),(5,10),(4,15)$, and $(5,15)$ had early warning responses to 5 dengue fever events, with the highest sensitivity and highest accuracy. This parameter combination has the strongest ability to discover dengue fever events. The combination of $(3,15)$ had early warning responses to 4 out of 5 dengue fever clustering events, with a sensitivity of $80 \%$; the $(3,5),(4,5)$, and $(5,5)$ combination had an early warning response to the dengue fever event with a sensitivity of $60 \%$ and the lowest accuracy. At the same time, a comparative analysis of the number of days of early warning for 9 parameter combinations was carried out. The $(3,10)$ combination can provide early warning in every dengue fever event, with an average of 2.6 days in advance. This parameter combination has the best early warning effect in the simulation warning. The combination of $(4,10),(5,10),(3,15)$, and $(5,15)$ can provide early warning in 4 dengue fever clusters, with an average of 2 days in advance. The combination 
of $(5,5)$ and $(4,15)$ can provide early warning in 3 dengue fever clusters, with an average of 1.6 days in advance. Comprehensive evaluation and analysis of sensitivity and early warning days found that the combination of $(3,10)$ has the best sensitivity and early warning day indicators, which can be used as the first choice for early warning parameters. The combination of $(4,10),(5,10)$, and $(5,15)$ has the best sensitivity, and the number of days of early warning is better, and it can be used as an alternative parameter combination.

\subsection{Use of continuous warning days for hierarchical warning analysis}

In our study, the average continuous warning days for 5 dengue fever clusters with 9 parameter combinations in descending order were $(3,10)>(4,10)=(5,10)=(5,15)>(3,15)>(4,15)=(3,5)>(4,5)=(5,5)$. At the same time, the preferred parameter combination obtained by the comprehensive evaluation and analysis of sensitivity and early warning days was $(3,10)$, and the alternative parameter combination was $(4,10),(5,10),(5,15)$, so it can also be considered. A graded warning response was made for the subsequent warning of dengue fever syndrome according to the continuous warning days of these 4 combinations. Since the number of early warning days for dengue fever set in this study does not exceed 4 days, the number of continuous warning days did not exceed 4 days. For example, if the combination of $(3,10)$ is used for early warning, when an early warning occurs for the first time, you can consider observing or closely observing this point (set as the first- or second-level response). If the early warning occurs continuously on the second day, one can consider on-site sampling and epidemiological investigation (set as the third level response). The result of this parameter combination can be compared with the warning results of the other 3 alternative parameters, and the specific implementation plan should be considered comprehensively to better determine the authenticity of the warning of dengue fever syndrome.

\section{Conclusion}

This study screened the time and space parameters of time-space rearrangement scan statistics for the early warning for dengue fever outbreaks. The following conclusions can be drawn.

4.1 Using the data of dengue fever syndrome, the spatiotemporal rearrangement scan of dengue fever events in Mengla County was carried out, and the optimal spatiotemporal aggregation combination was selected. In the future, when using dengue fever syndrome to warn of dengue outbreaks in this area, priority can be given to the parameter combination with a maximum time cluster of 3 days and a maximum radius of 10 kilometres; in addition, the maximum time cluster is 4 days, and the maximum radius is 10 days; the maximum time cluster is 5 days, and the largest radius is 10 days; the maximum time cluster is 5 days, and the maximum radius is 15 days can be used as an alternative parameter combination.

4.2 In the future use of optimal parameter combinations for dengue fever early warning, hierarchical responses can be adopted according to actual conditions. To ensure that the true aggregation event is detected to the greatest extent, it also avoids excessive waste of resources. 


\section{Declarations}

\section{Ethical Approval and Consent to participate}

Not applicable.

\section{Consent for publication}

All authors agree to publish.

\section{Availability of supporting data}

Not applicable.

\section{Competing interests}

The authors declare no Conflict of Interest.

\section{Funding}

Research and Application of Symptom Monitoring and Early Warning Prevention and Control System in the Border Areas of Yunnan Province(214YNPHXT23);

National Nature Science Foundation of China (81860607);

Innovative Research Team of Yunnan Province (2019(6)), China.

\section{Authors' contributions}

Rongyan Gu:Data analysis, article writing, literature review,methodology

Shen Huang:Article writing, data collection, article revision,article structure

Ling Zhang:Data collection

Wei Liu:Funding, providing resources

Yan Li:Funding, providing resources

\section{Acknowledgements}

This work is jointly supported by Research and Application of Symptom Monitoring and Early Warning Prevention and Control System in the Border Areas of Yunnan Province(214YNPHXT23), National Nature Science Foundation of China (81860607) and Innovative Research Team of Yunnan Province (2019(6)), China.

\section{Authors' information}


Rongyan Gu M.D

School of Public Health, Kunming Medical University

1737179823@qg.com,18487338287(c)

Shen Huang M.D

School of Public Health, Kunming Medical University

huangshen721@163.com,15108668637(c)

Ling ZhangM.D

School of Public Health, Kunming Medical University

1206694587@qg.com,18788486993(c)

Liu Wei PhD Prof.

School of Public Health, Kunming Medical University

liuweikm@qq.com, 18388245021(c)

Yan Li PhD Prof.

School of Public Health, Kunming Medical University

yanli20021965@21cn.com

\section{References}

1. Mou D, He YX, Chen QL, et al. Comparison of epidemiological features between imported and indigenous dengue fever cases in China[J]. Disease Surveillance. 2017;32(03):184-9.

2. Li YY, Zhu J, Mi PF, et al. Analysis of situation of awareness and behavior forming on dengue in Xishuangbanna[J]. Chinese Journal of Public Health Management. 2017;33(01):86-8.

3. WHO. Global strategy for dengue Prevention and control.Geneva,World Health Organization,2012 [EB/OL].

4. Li YY, Zhu J, Li HB. Distribution of the dengue fever vector in Xishuangbanna Prefecture of Yunnan[J]. China Tropical Medicine. 2016;16(03):237-9 + 265.

5. Li HX, Zhou HN, Yang YC, Jiang H. Dengue fever epidemic situation in Yunnan province from 2004 to 2008[J]. Chinese Journal of Vector Biology Control. 2010;21(06):576-7 + 580.

6. Kulldorff M. ProsPective Time Periodic GeograPhical Disease Surveillance Using a Scan Statistic[J]. J Roy Stat Soc. 2010;164(1):61-72. 
7. Xie Y, Chongsuvivatwong V, Tang Z. SPatio-TemPoral Clustering of Hand, Foot, and Mouth Disease at the County Level in Guangxi, China[J]. Plos One. 2014;9(2):e88065.

8. Tang F, Cheng Y, Bao C. SPatio-temPoral trends and risk factors for Shigella from 2001 to 2011 in Jiangsu Province, PeoPle's RePublic of China.[J]. Plos One. 2014;9(1):e83487.

9. Kulldorff M, Heffernan R, Hartman J. A SPace-Time Permutation Scan Statistic for Disease Outbreak Detection[J]. Plos Medicine. 2005;2(3):e59.

10. Li Y, Zhang HY, Zhang XD, et al. Comparative study on clustering in hand foot and mouth disease based on spatial and temporal scanning analysis and circular distribution[J]. Disease Surveillance. 2016;31(08):638-41.

11. Pei J, Ma Y, Zhang XY, et al. Study on the baseline selection of the prospective space-time scan statistic model in early warning of infectious diseases[J]. Modern Preventive Medicine. 2012;39(09):2131-5.

12. Ai LY, Chen EF, Sun JM, et al. Analysis on spatial and temporal clustering of dengue fever epidemic in Zhejiang, 2004 - 2016[J]. Disease Surveillance. 2019;34(01):27-31.

13. Tang XY, Li Q, Chou XQ, et al. An exploratory study on maximum spatial cluster size of scan statistics[J]. Chinese Journal of Disease Control Prevention. 2015;19(03):316-7 + 320 .

14. Coulston JW, Riitters KH. GeograPhic analysis of forest health Indicators using sPatial scan statistics[J].Environ Manag,2003,31(6):764-767.

15. Xiao YY. A Dissertation Submitted in Partial Fulfillment of the Requirements for the Degree of Master in Medicine[D].Wuhan University of Science and Technology,2015.

16. Wang MY. The Establishment of Syndromic Surveillance in Medical Institutions of Mengla County and the Study on the Quality of its Data[D]. Kunming Medical University, 2017.

17. Coleman M, Coleman M, Mabuza AM. Using the SaTScan method to detect local malaria clusters for guiding malaria control Programmes[J]. Malaria Journal. 2009;8(1):68.

18. Tang JB, Deng M, Liu QL. On Spatio-temporal Events Clustering Methods[J]. Geomatics World. 2013;20(01):38-45.

19. Zhao D. Study on Early Warning Technique of Dengue Fever Based on Imported Cases, Vectors and Climate Factors[D]. Chinese Center for Disease Control and Prevention, 2012.

20. Zhang F, Song YL, Zhang Y, et al. Establishment of symptom surveillance and early warning system in border area in Yunnan[J]. Disease Surveillance. 2016;31(11):903-8.

21. Chen W, Wu SG, Ou JM, et al. Application of fever symptom monitoring in prospective early outbreak of dengue fever[J]. Strait Journal of Preventive Medicine. 2018;24(02):4-7.

\section{Figures}




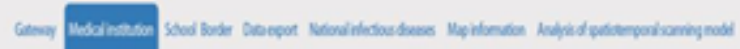

Please select the fime interval, location and symptom type you want to queny.

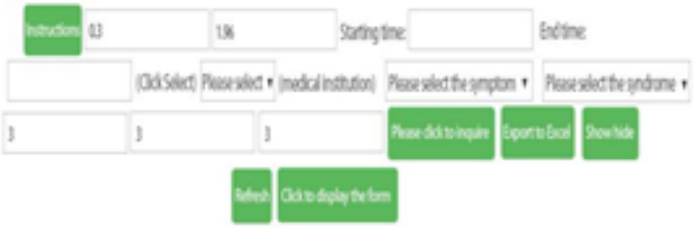

Symptom detection system in Yunnan border area

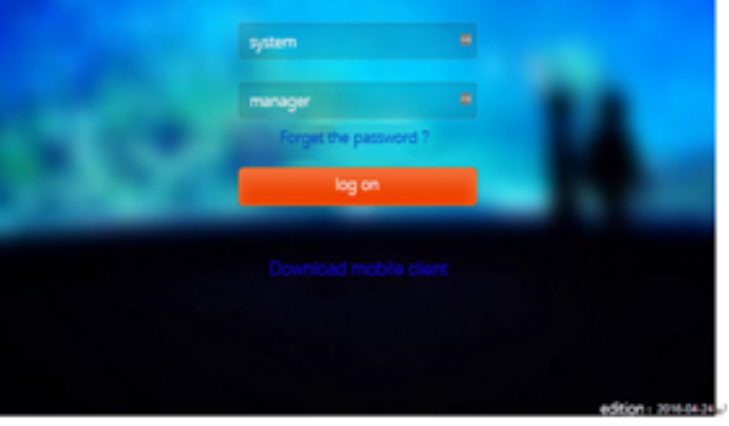

Figure 1

Login interface 


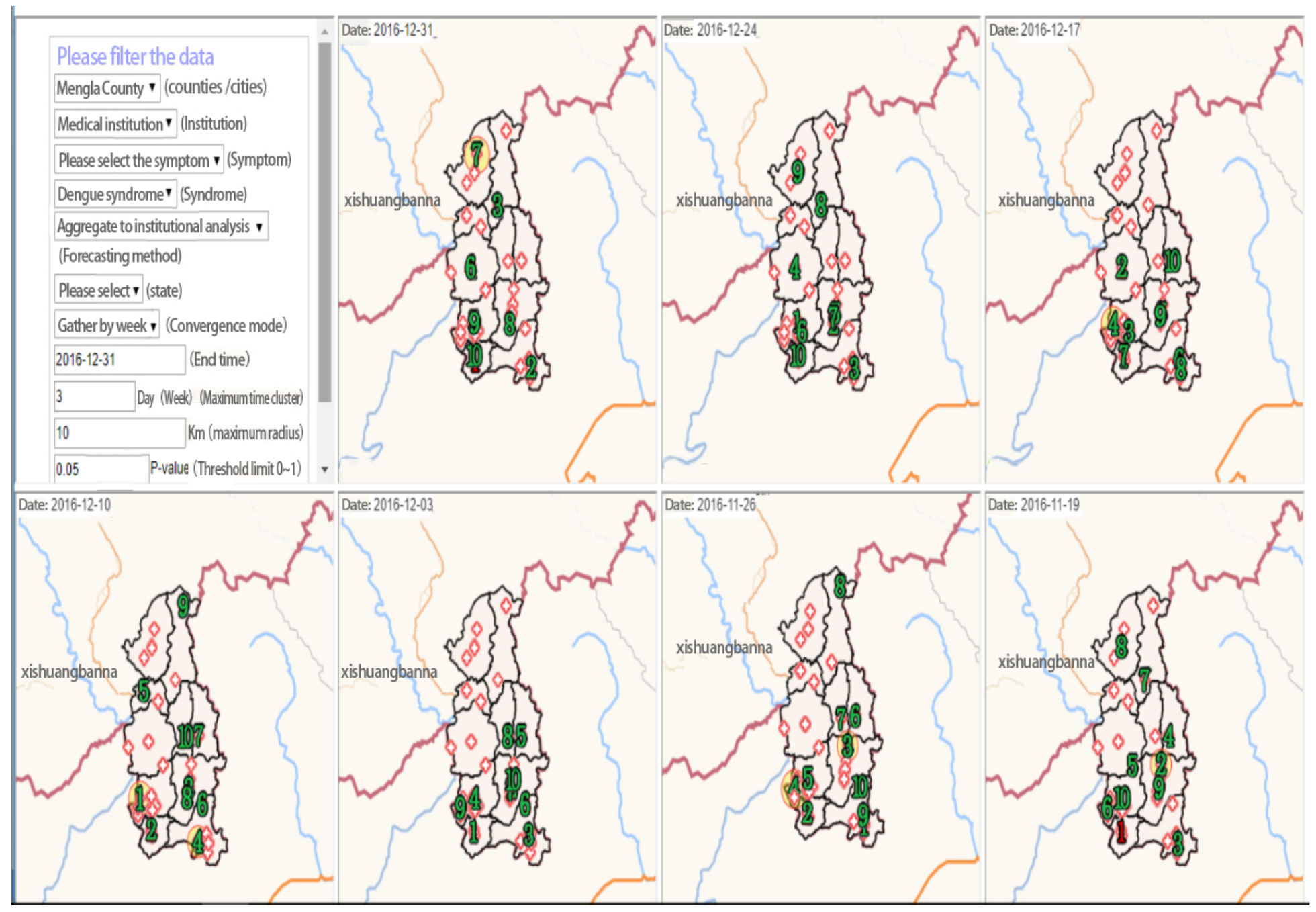

Figure 2

Data selection dialogue box interface 


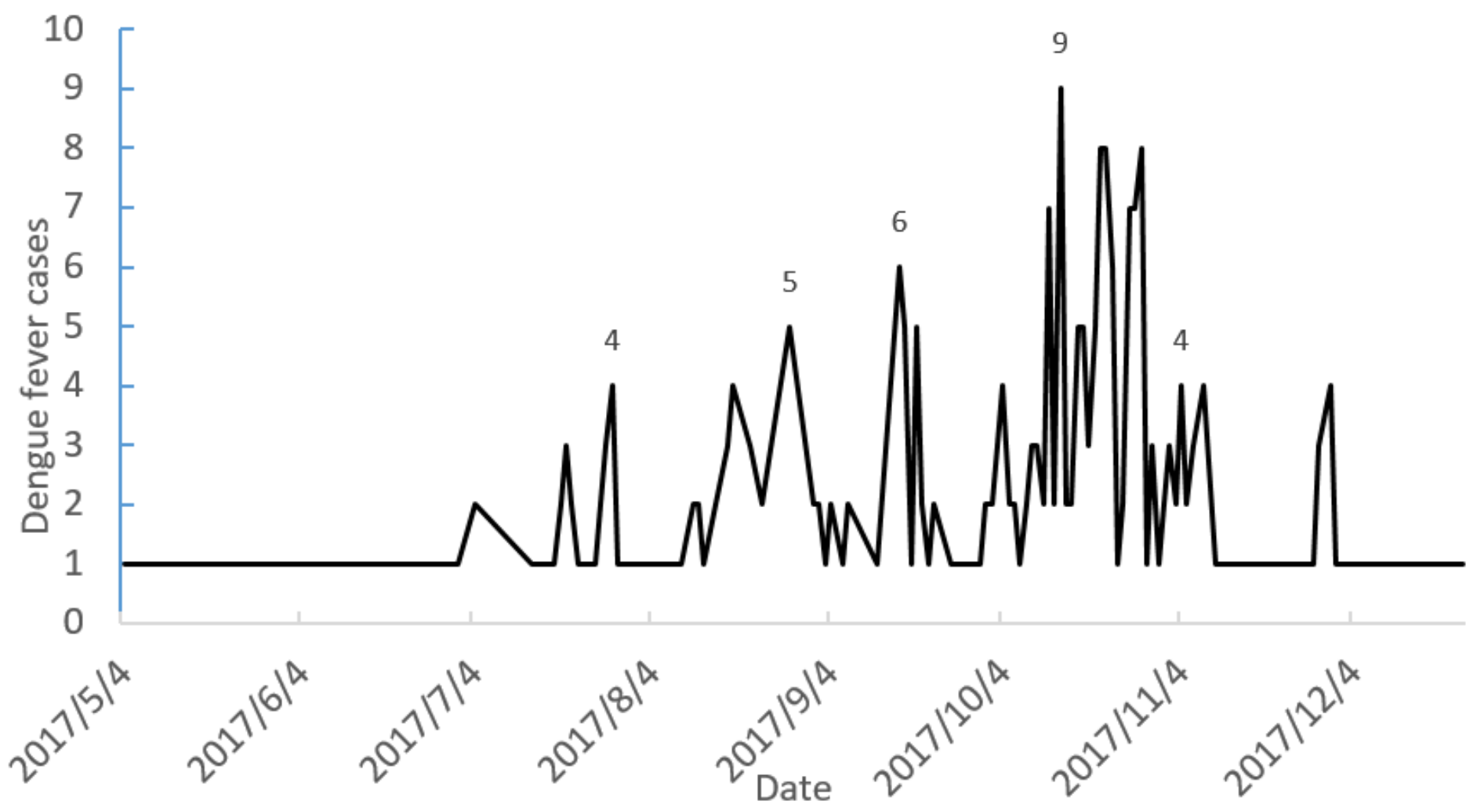

Figure 3

Daily distribution of dengue fever cases 

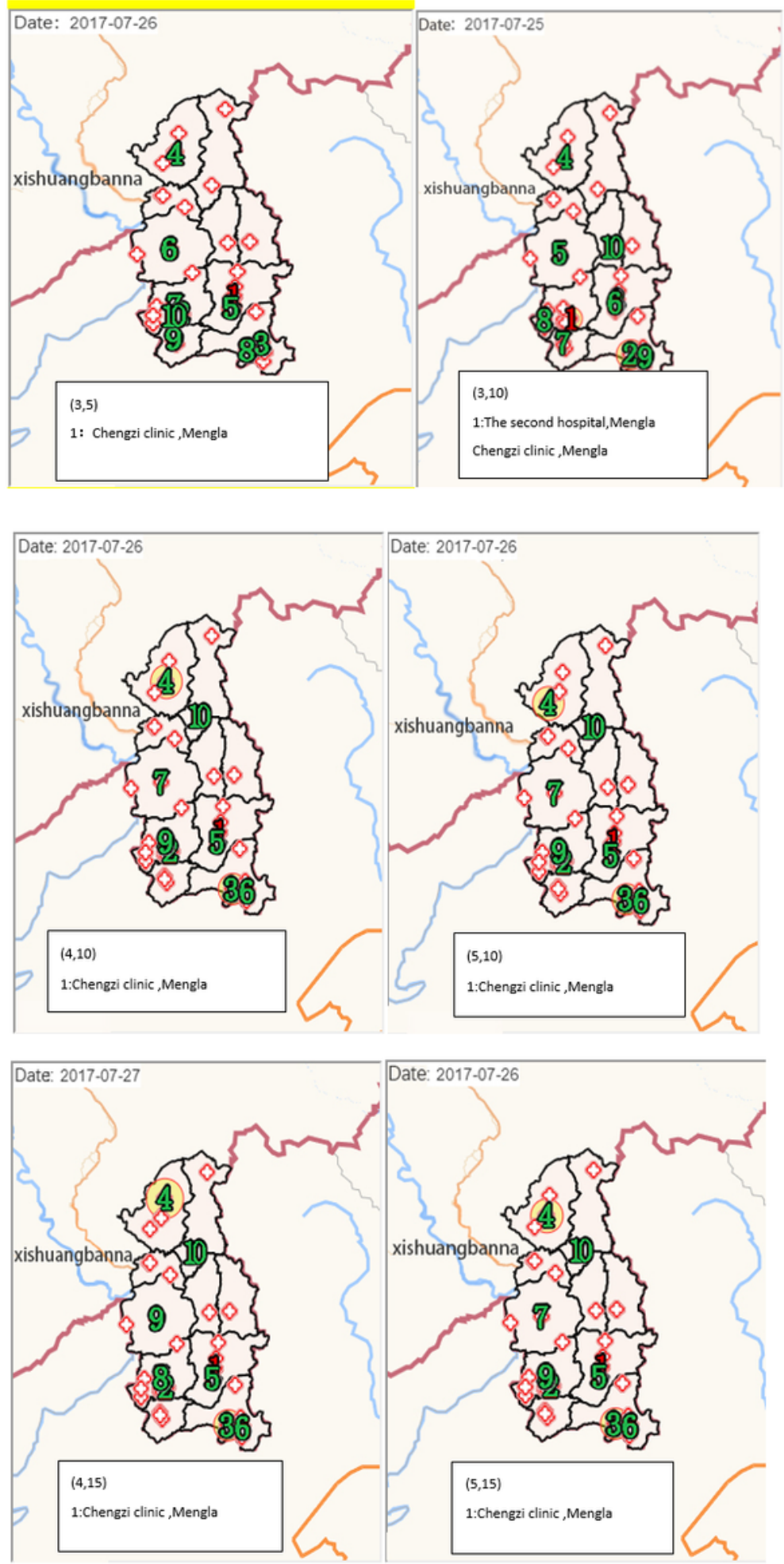

\section{Figure 4}

The first case of dengue fever aggregation event was scanned with different spatiotemporal parameters of dengue syndrome 
Tise and space scan map of the reasining 4 dengue fever eluster
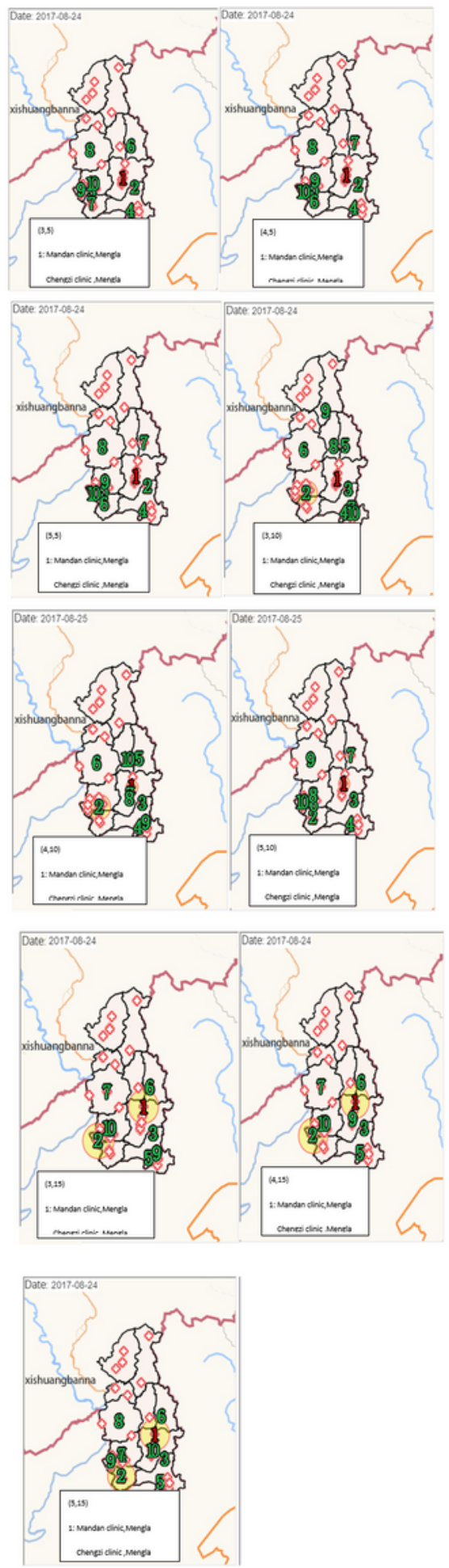

Figure 5

The second dengue fever gathering incident uses a combination scan of different time and space parameters of the dengue fever syndrome 

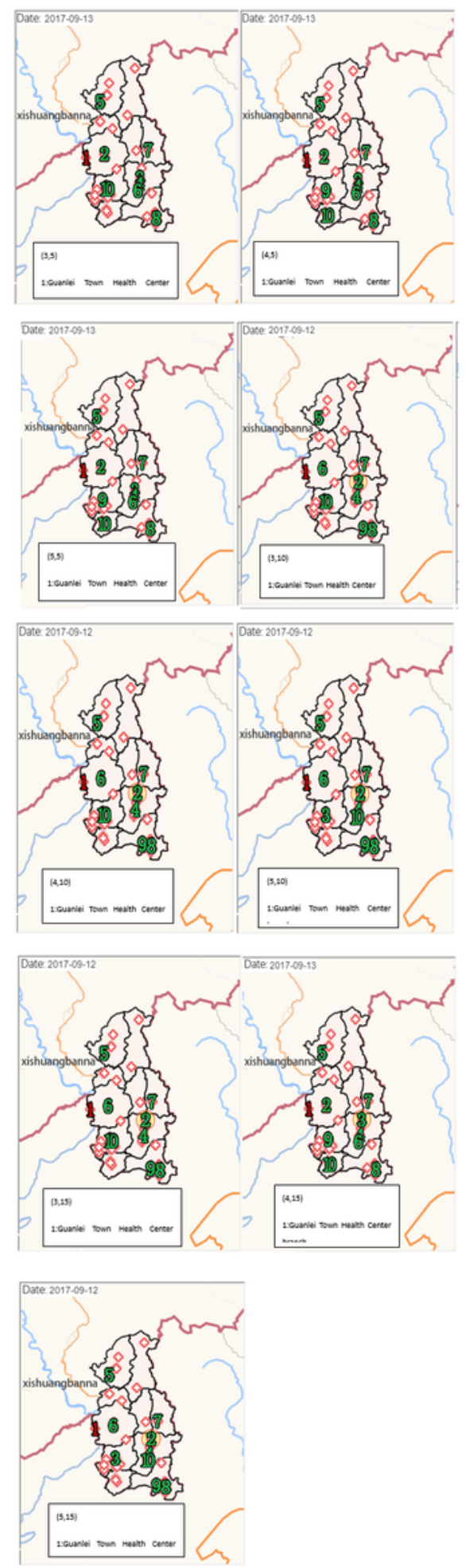

\section{Figure 6}

The third dengue fever gathering incident uses a combination scan of different time and space parameters of the dengue fever syndrome 


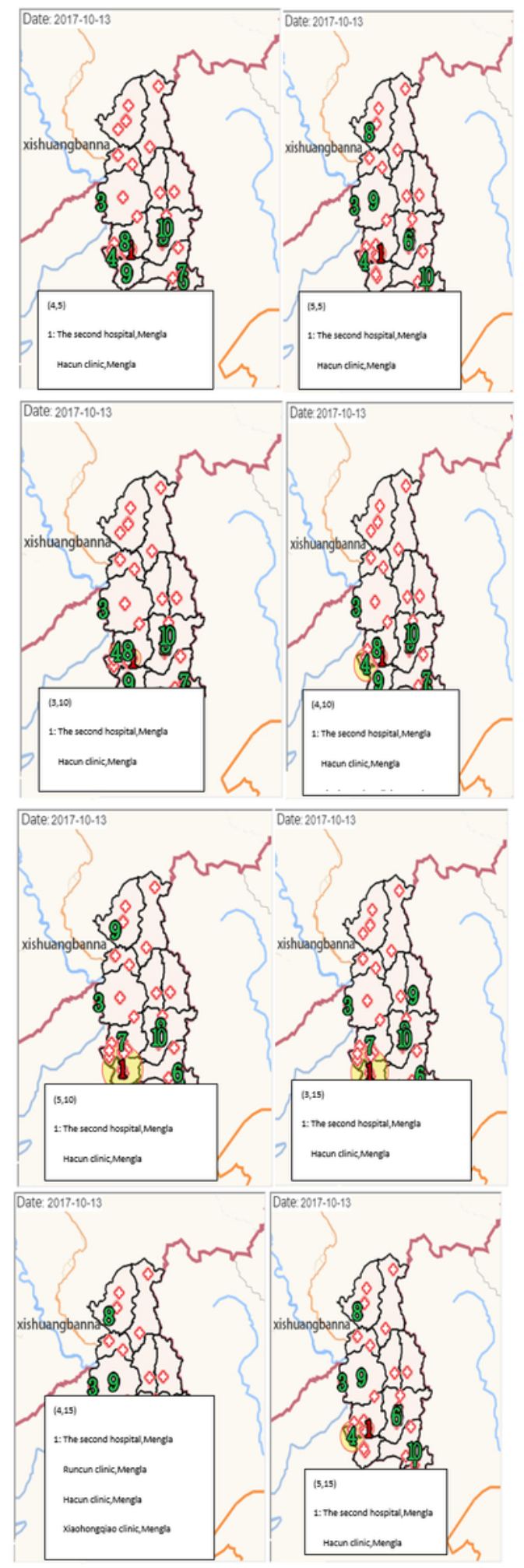

\section{Figure 7}

The forth dengue fever gathering incident uses a combination scan of different time and space parameters of the dengue fever syndrome 

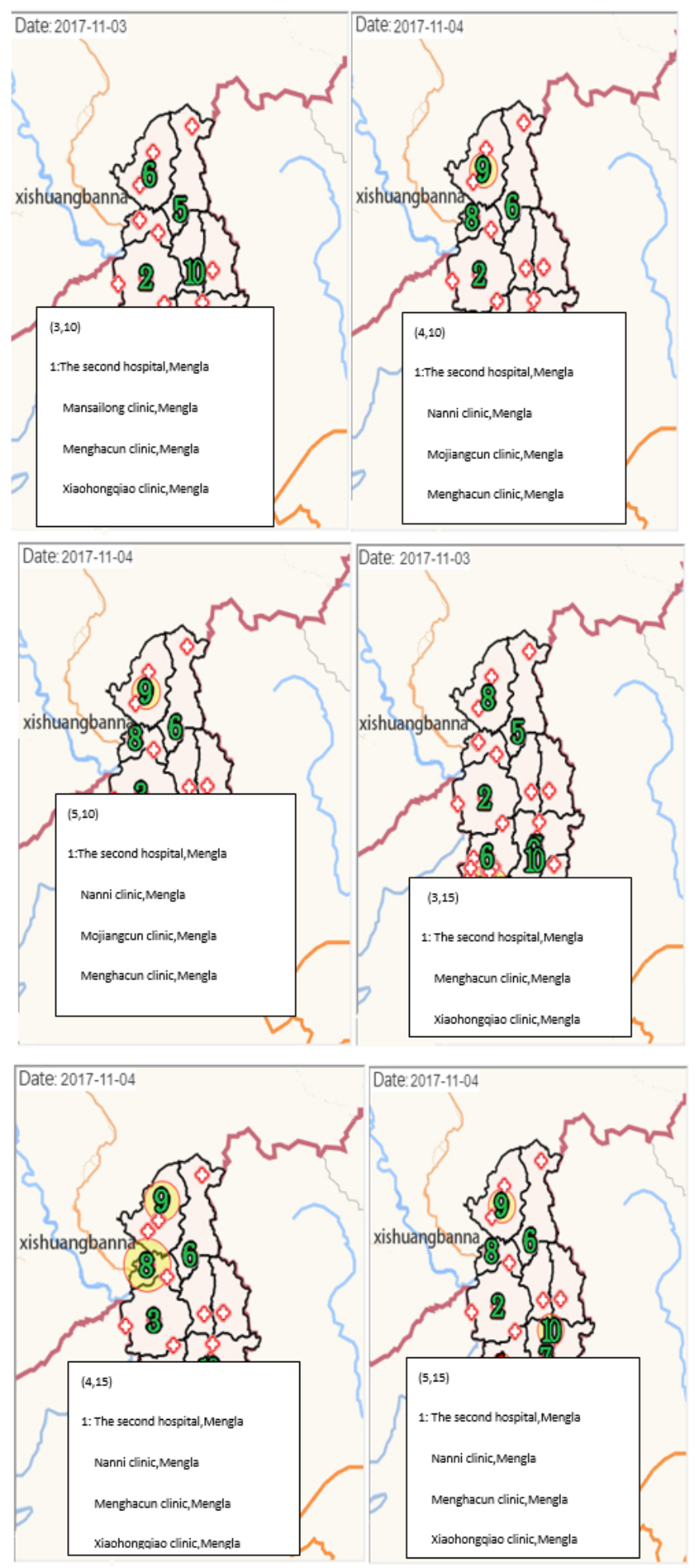

\section{Figure 8}

The fifth dengue fever gathering incident uses a combination scan of different time and space parameters of the dengue fever syndrome 